\title{
Security Group
}

National Cancer Institute

\section{Source}

National Cancer Institute. Security Group. NCI Thesaurus. Code C44271.

A group created to indicate the access level of member individuals. 